\title{
Hospital Readmission After Climbing-Related Injury in the United States
}

\author{
Joseph D. Forrester, MD, MSc; Kristin A. Hunter, MD; Lakshika Tennakoon, MPhil, MSc; David A. Spain, MD \\ Division of General Surgery, Department of Surgery, Stanford University, Stanford, CA
}

\begin{abstract}
Introduction-Rock climbing and mountaineering may result in injury requiring hospital admission. Readmission frequency after climbing-related injury is unknown. The aim of this study was to assess readmission frequency, morbidity, and mortality after admission for climbing-related injury.

Methods-We performed a retrospective analysis of the 2012 to 2014 national readmission database, a nationally representative sample of all hospitalized patients. Rock climbing, mountain climbing, and wall climbing injuries were identified using International Classification of Diseases-Ninth Revision-Clinical Modification codes (E004.0). Outcomes evaluated included readmission frequency, morbidity, mortality, inpatient admission, and costs. Adjusted analyses accounting for survey methodology were performed. Data are presented as mean \pm SD.

Results-A weighted-estimate 1324 inpatient admissions were associated with a climbing-related injury. Most patients were aged 18 to 44 y $(64 \%)$, and $68 \%(n=896)$ were male. Isolated extremity injures were more common than other injuries $(49 \%, n=645)$. Sixty-five percent $(n=856)$ underwent a major operative procedure. Less than $1 \%$ of all climbing-related visits resulted in death. Within 6 mo of the index hospitalization, $2 \%(n=23)$ of the patients had at least 1 readmission, with a time to readmission of $9.9 \pm 6.6(95 \% \mathrm{CI}$ 4.5-15.4) d. Only female sex was associated with increased odds of readmission (odds ratio $=5.5 ; 95 \% \mathrm{CI}$ $1.5-20.1 ; P=0.01)$.

Conclusions - There is a very low frequency of readmissions after being admitted to the hospital for climbing-related injury. A considerable opportunity to describe the long-term burden of climbing-related injury exists, and further research should be done to assess injury burden treated in the outpatient setting.
\end{abstract}

Keywords: trauma, climbing, rock climbing, morbidity, mortality, injury burden

\section{Introduction}

Rock climbing, mountaineering, and indoor rock climbing have become increasingly popular nationwide over the past few decades. ${ }^{1-3}$ It is estimated that there are over 6 million active participants in indoor and outdoor climbing in the United States alone. ${ }^{1}$ With an $8 \%$ growth in the number of climbing gyms opened per year over the past $5 \mathrm{y}$, climbing is also more accessible to the general population. ${ }^{1}$ Climbing-related injury, although uncommon, can be associated with considerable morbidity, with a hospital

Corresponding author: Joseph D. Forrester, MD, MSc, Stanford University, Department of Surgery, 300 Pasteur Drive, H3591, Stanford, CA 94305; e-mail: jdf1@ stanford.edu.

Submitted for publication January 2020.

Accepted for publication May 2020. admission rate after emergency department presentation of $11 \% .^{1,4}$

A range of injuries can occur during climbing, from inherent risk of falling to stress-related musculoskeletal injuries. ${ }^{1,5}$ Up to $60 \%$ of persons experiencing climbingrelated injury have multiple body regions injured, and nearly $45 \%$ of injured climbers report some type of longterm disability. ${ }^{1,6}$ Despite this potential for disability, little is known about the morbidity associated with climbingrelated injury. ${ }^{2,3}$ This study aims to assess the readmission frequency, morbidity, and mortality after admission for climbing-related injury.

\section{Methods}

The study was classified as exempt after a Stanford University institutional review board assessment because it 
contained no identifiable data. We used data from the agency for healthcare and quality, heathcare cost and utilization project (HCUP), and national readmission database (NRD) 2012 to 2014 to describe the epidemiology of inpatient climbing-related injury in the United States. ${ }^{7}$ NRD is the largest all-payer readmission database in the United States, capturing both inpatient encounters that result in discharge or transfer and those that result in readmission. ${ }^{7}$ Publicly available, NRD is constructed from the HCUP state inpatients databases. ${ }^{7}$ When analyzed, NRD is weighted to provide national estimates on the roughly 107 million discharges in the United States between 2012 to $2014 .^{8}$ The number of hospitals included in the sample ranged from 1715 to $2048 .{ }^{8}$ We included all inpatient encounters with the diagnosis code corresponding to rock climbing, mountain climbing, or wall climbing injury (International Classification of Disease [ICD], 9th Revision, Clinical Modification [CM] code E004.0).

Rock climbing, mountaineering, and wall climbing injuries were collectively analyzed as "climbing-related injuries" because no further specificity is available in the NRD dataset. Variables assessed included age, sex, payer status, patient household income quartile by zip code, anatomic location of injury, injury severity score (ISS), length of stay, need for a major operative procedure, discharge destination, hospitalization cost, need for readmission after index admission, and time to readmission. An index admission was defined as the first hospital admission a patient had over the observed time period. A major operative procedure was pre-defined by HCUP in accordance with diagnosis-related groups. ${ }^{7}$ Costs were derived from reported charges converted to costs using HCUP cost-tocharge ratios. Injury characteristics were further evaluated using the Stata-based ICD programs for injury categorization module. ${ }^{9}$ ISS is a validated anatomic scoring system that provides a measure of the overall injury severity in the injured patient. ${ }^{10,11}$ Intensive care unit level of care was defined as need for invasive ventilation or vasopressors. The 2012 to 2014 time period was chosen because these databases were both available at the time of analysis and had consistent ICD-9 coding to enable combinatorial analysis.

Outcomes of interest included readmission frequency, morbidity, mortality, inpatient admission, and cost. All statistical analyses used NRD sampling strata and discharge weights to produce nationally weighted patient-level estimates and $95 \%$ CIs that account for clustering of patients among hospitals. Weighted values are reported unless otherwise specified. Data are presented as mean \pm SD (range), as appropriate; calculated weighted means are reported with $95 \%$ CI according to survey statistical methodology. ${ }^{8}$ The Fisher exact and Wilcoxon rank sum test were used as appropriate. Odds ratios (ORs) with
95\% CIs are reported as outputs from the multivariate analysis. The a priori $P$-value considered significant was $P<0.05$. Stata 12.0 (StataCorp, College Station, TX) was used for all statistical analyses.

\section{Results}

There were a total of 600 unweighted adult inpatient admissions associated with a climbing-related injury, with 200 \pm 36 (163-235) climbing-related injuries per year. After weighting, the estimate was a total of 1324 inpatient admissions over the observed time period, with $441 \pm 61$ climbing-related injuries resulting in admission annually (Table 1). Patient age was $31 \pm 15(4-84)$ y, and $896(62 \%)$ were male. Most injured patients $(n=808$, $61 \%$ ) had private insurance and were in the upper half of the income quartile of those living in their zip code. Among those admitted, isolated extremity injuries $(49 \%, \mathrm{n}=645)$ and multiple body system injuries $(37 \%$, $\mathrm{n}=488)$ were common. Only $13 \%(\mathrm{n}=175)$ patients had an ISS $>15$, and $65 \%(n=856)$ underwent an operative procedure.

The most common operative procedure during an index admission was open reduction and internal fixation (ORIF) of a tibia or fibula $(n=299)$, which accounted for $35 \%$ of major operative procedures performed. Other common surgical procedures included vertebral fracture repair or fusion (13\%, $\mathrm{n}=108$ [including ICD-9 CM codes 0353,8162 , and 8163]), ORIF of tarsals or metatarsals $(11 \%, \mathrm{n}=98)$, and ORIF of the radius or ulna $(10 \%, n=88)$. Length of stay was $4.6 \pm 7.3 \mathrm{~d}$, and most patients were discharged to home $(83 \%, \mathrm{n}=1105)$. Less than $1 \%$ of all climbing-related admissions resulted in death. Total cost for all initial admissions was $\$ 26,200,000$.

There were 23 (2\%) patients readmitted after their index operation, with a time to readmission of $9.9 \pm 6.6(95 \% \mathrm{CI}$ 4.5-15.4) d. Most (70\%, $\mathrm{n}=16)$ readmitted patients were female. The only demographic variable that was statistically significantly different between those patients who were readmitted and those who were not was female sex $(\mathrm{OR}=5.5 ; 95 \%$ CI $1.5-20.1 ; P=0.01)$.

\section{Discussion}

The popularity of participating in rock-climbing, mountaineering, and indoor wall climbing has increased considerably over the past decades; as such, climbing-related injury has also increased. ${ }^{1}$ Although several prior studies have delineated admission frequency, anatomic distribution of injury, and inpatient mortality, there are scant data describing the broader burden of climbing-related injury. To help describe this burden in greater detail, we explored the frequency of readmission among patients who 
Table 1. Epidemiology of patients readmitted after climbing-related injury in the United States-2012 to 2014

\begin{tabular}{|c|c|c|c|c|}
\hline & All patients & $\begin{array}{l}\text { Patients not } \\
\text { readmitted }\end{array}$ & $\begin{array}{l}\text { Patients readmitted } \\
\text { at least once within } \\
6 \text { mo of discharge }\end{array}$ & P-value \\
\hline No. & 1324 & $1301(98)$ & $23(2)$ & - \\
\hline \multicolumn{5}{|l|}{ Age, n (\%) } \\
\hline$<18 \mathrm{y}$ & $229(17)$ & $229(18)$ & - & \multirow[t]{5}{*}{0.9} \\
\hline $18-44 \mathrm{y}$ & $848(64)$ & $828(64)$ & $20(87)$ & \\
\hline $45-64$ y & $203(15)$ & $200(15)$ & $<10^{\mathrm{a}}$ & \\
\hline $65-74 y$ & $39(3)$ & $39(3)$ & - & \\
\hline $75-84 y$ & $<10^{\mathrm{a}}$ & $<10^{\mathrm{a}}$ & - & \\
\hline Male sex, n (\%) & $896(68)$ & $890(68)$ & $<10^{\mathrm{a}}$ & 0.05 \\
\hline \multicolumn{5}{|l|}{ Payment data, n (\%) } \\
\hline Medicare & $62(5)$ & $62(5)$ & - & \multirow[t]{6}{*}{0.5} \\
\hline Medicaid & $173(13)$ & $167(13)$ & $<10^{\mathrm{a}}$ & \\
\hline Private insurance & $836(63)$ & $822(63)$ & $14(60)$ & \\
\hline Self-pay & $129(10)$ & $126(10)$ & $<10^{\mathrm{a}}$ & \\
\hline No charge & $23(2)$ & $23(2)$ & - & \\
\hline Other & $102(8)$ & $102(8)$ & - & \\
\hline \multicolumn{5}{|l|}{$\begin{array}{l}\text { Household income compared to others in } \\
\text { patient's zip code (quartile), n (\%) }\end{array}$} \\
\hline $0-25$ th & $216(16)$ & $213(16)$ & $<10^{\mathrm{a}}$ & \multirow[t]{4}{*}{0.6} \\
\hline 26th-50th & $301(23)$ & $293(22)$ & $<10^{\mathrm{a}}$ & \\
\hline $51 \mathrm{st}-75 \mathrm{th}$ & $363(27)$ & $355(27)$ & $<10^{\mathrm{a}}$ & \\
\hline 76th-100th & $445(34)$ & $440(34)$ & $<10^{\mathrm{a}}$ & \\
\hline \multicolumn{5}{|l|}{ Anatomic site of injury, $n(\%)$} \\
\hline Isolated head/neck & $55(4)$ & $55(4)$ & - & \multirow[t]{7}{*}{0.7} \\
\hline Isolated face & $<10^{\mathrm{a}}$ & $<10^{\mathrm{a}}$ & - & \\
\hline Isolated chest & $48(4)$ & $46(4)$ & $<10^{\mathrm{a}}$ & \\
\hline Isolated abdomen & $79(6)$ & $79(6)$ & - & \\
\hline Isolated extremity & $645(49)$ & $628(48)$ & $17(73)$ & \\
\hline Isolated external & $<10^{\mathrm{a}}$ & $<10^{\mathrm{a}}$ & - & \\
\hline Multiple body regions & $488(37)$ & $483(37)$ & $<10^{\mathrm{a}}$ & \\
\hline Injury severity score $>15, \mathrm{n}(\%)$ & $175(13)$ & $170(13)$ & $<10^{\mathrm{a}}$ & 0.2 \\
\hline Underwent major operative procedure, n (\%) & $856(65)$ & $847(65)$ & $<10^{\mathrm{a}}$ & 0.2 \\
\hline $\begin{array}{l}\text { Length of stay at initial operation, } \\
\text { mean } \pm \text { SD }[95 \% \mathrm{CI}], \mathrm{d}\end{array}$ & $4.6 \pm 7.3[4.1-5.2]$ & $4.7 \pm 7.4[4.1-5.3]$ & $2.5 \pm 1.4[1.7-3.2]$ & 0.3 \\
\hline ICU-level care, $\mathbf{n}(\%)$ & $18(1)$ & $18(1)$ & - & 1.0 \\
\hline \multicolumn{5}{|l|}{ Discharge destination, n (\%) } \\
\hline Died in hospital & $<10^{\mathrm{a}}$ & $<10^{\mathrm{a}}$ & - & \multirow[t]{6}{*}{0.8} \\
\hline Home & $1105(83)$ & $1084(83)$ & $21(91)$ & \\
\hline Transfer to another facility & $19(1)$ & $19(1)$ & - & \\
\hline Skilled nursing facility & $101(8)$ & $101(8)$ & - & \\
\hline Home health & $82(6)$ & $80(6)$ & $<10^{\mathrm{a}}$ & \\
\hline Against medical advice & $10(1)$ & $10(1)$ & - & \\
\hline Days to readmission, mean \pm SD $[95 \% \mathrm{CI}]$ & $9.9 \pm 6.6[4.5-15.4]$ & - & $9.9 \pm 6.6[4.5-15.4]$ & N/A \\
\hline
\end{tabular}

N/A, not applicable.

${ }^{\mathrm{a}}$ Values $<10$ are not reported in accordance with HCUP data use agreement. 
experienced climbing-related injury in the United States. Reassuringly, readmission frequency is quite low. Only $2 \%$ of patients were readmitted within 6 mo of discharge. This percentage compares favorably to trauma patients as a whole. A prior study analyzing 30-d readmission for all trauma patients found a readmission rate of $<3 \%$; thus, the readmission rate after climbing injury is on par with that among other trauma patients. ${ }^{12}$ Compared to other mechanisms, climbing-related injury readmission rates were low-gunshot wounds had the highest rate of readmission at $11 \%$, followed by motorcycle crash victims at $4 \%{ }^{12}$

The only demographic variable that showed statistical significance was female sex. There are several possible explanations for this finding. It is possible that men tend to not seek healthcare as often as women, with women self-reporting a greater number of visits to their doctor for both physical and mental health reasons. ${ }^{13}$ Another possible explanation has to do with mechanical factors specific to climbing. For example, some climbing shoes are designed for the male ankle, resulting in higher stress on the female Achilles tendon. ${ }^{3}$ In addition, another study has suggested that women may be more predisposed to chronic climbing injuries such as carpal tunnel syndrome. ${ }^{3}$ Having acute or chronic injury from climbing could increase the chances of readmission, although this level of granular detail about pre-existing chronic injuries was not captured in our study. ${ }^{3}$ A study from 2017 correlates with our findings, noting that female patients were statistically more significantly likely $(P<0.01)$ to be readmitted after any trauma. ${ }^{14}$

There was a high incidence of orthopedic injuries among admitted and readmitted climbers, with isolated extremity injury accounting for $49 \%$ of initial admissions and $73 \%$ of readmissions. Several other studies have reported similar findings in that fractures, sprains, and strains account for a large portion of injury. ${ }^{1,4,15}$ The high frequency can be explained by the natural force and stress put on the body's musculoskeletal system during climbing, resulting in high risk for mechanical injury. ${ }^{1}$ In an orthopedic trauma study from 2019, the readmission rate after orthopedic trauma was 5\%; one-third of these readmissions were due to direct orthopedic complications. ${ }^{16}$ Climbers may, at first glance, seem well equipped to deal with isolated extremity injuries because recreational climbers are commonly active at baseline. Healthcare providers could be biased and assume these patients may not require long-term or higher level of care at discharge. These patients may be discharged without adequate home support, physical therapy, and follow-up, resulting in more frequent readmission.

The longer-term morbidity of climbing-related injury once a patient has been discharged from a hospital remains incompletely described. A study from 2017 suggests that climbing-related injury results in long-term disability nearly $45 \%$ of the time. ${ }^{6}$ In our prior investigation of the national emergency department sample, nearly one-fifth of patients admitted for climbing-related injuries are discharged to a skilled nursing facility or with home health services, suggesting a considerable burden of injury. ${ }^{1}$ The trauma associated with the injury may also increase susceptibility to psychological distress. ${ }^{6}$ The extent of the burden resulting from climbing-related injury needs to be further quantified.

This study has limitations. The inherent potential for injury in climbing occurs along a spectrum depending on the climbing discipline being practiced, and this complexity is not characterized in the NRD dataset. Second, there may be misclassification bias associated with using ICD-9 codes, and climbing-related injury may not even be captured in the severely injured poly-trauma patient. Third, because no universally accepted definitions exist for rock climbing, wall climbing, and mountaineering, reporting of activities not typically associated with climbing may have been included inadvertently by coders, falsely increasing the number of recreational climbing-related injuries. Fourth, our study only captures persons initially admitted as inpatients; we would not have captured a patient who initially presented to an outpatient physician, only to subsequently require hospital admission. Similarly, this study would not capture patients who died before or during transport. Fifth, NRD is an annual file containing patients discharged in a calendar year; a patient admitted in the latter part of the year may not be captured if the readmission crossed into the next year. Approximately $1 \%$ of admissions are expected to be missing as a result of this reporting feature. ${ }^{8}$ Sixth, patients initially admitted in 1 state and readmitted in a separate state are not linked in NRD, which may artificially lower the readmission frequency. This may be particularly pronounced when climbers travel to other states for highly sought climbing destinations. Finally, NRD is a survey in which regional selection bias could lead to overestimation or underestimation of climbing-related injury.

\section{Conclusions}

There is a very low frequency of readmissions after admission to the hospital for climbing-related injury. Readmission frequency was statistically significantly higher in female patients, which may be multifactorial. Considerable opportunity remains to describe the long-term burden of climbing-related injury, and further research should be 
done to assess injury burden treated in the outpatient setting.

Author Contributions: Writing, data analysis, data interpretation (JDF); writing, data analysis, data interpretation $(\mathrm{KH})$; data collection, data analysis, critical review (LT); writing, critical review (DS); final approval of manuscript (JDF, KAH, LT, DAS).

Financial/Material Support: None.

Disclosures: None.

\section{References}

1. Forrester JD, Tran K, Tennakoon L, Staudenmayer K. Climbing-related injury among adults in the United States: 5-year analysis of the National Emergency Department Sample. Wilderness Environ Med. 2018;29(4):425-30.

2. Gronhaug G, Saeterbakken A. No pain no gain: a survey of use of healthcare and reasons not to seek healthcare by Norwegian climbers with chronic injuries. BMJ Open Sport Exerc Med. 2019;5(1), e000513.

3. Gronhaug G. Self-reported chronic injuries in climbing: who gets injured when? BMJ Open Sport Exerc Med. 2018;4(1), e000406.

4. Nelson NG, McKenzie LB. Rock climbing injuries treated in emergency departments in the US, 1990-2007. Am J Prev Med. 2009;37(3):195-200.

5. Buzzacott P, Schoffl I, Chimiak J, Schoffl V. Rock climbing injuries treated in US emergency departments, 2008-2016. Wilderness Environ Med. 2019;30(2):121-8.

6. McDonald JW, Henrie AM, Teramoto M, Medina E, Willick SE. Descriptive epidemiology, medical evaluation, and outcomes of rock climbing injuries. Wilderness Environ Med. 2017;28(3):185-96.

7. Healthcare Cost and Utilization Project. NRD Overview. Available at: https://www.hcup-us.ahrq.gov/nrdoverview. jsp. Accessed January 22, 2020.
8. Healthcare Cost and Utilization Project. Introduction to the HCUP Nationwide Readmissions Database (NRD) 2010-2016. Available at: https://www.hcup-us.ahrq.gov/db/ nation/nrd/Introduction_NRD_2010-2016.pdf. Accessed September 5, 2019.

9. Clark DE, Osler TM, Hahn DR. ICD Programs for Injury Categorization (ICDPIC), Version 3.0. 2010. http://ideas. repec.org/c/boc/bocode/s457028.html. Accessed July 13 2020.

10. Boyd CR, Tolson MA, Copes WS. Evaluating trauma care: the TRISS method. Trauma Score and the Injury Severity Score. J Trauma. 1987;27(4):370-8.

11. Glance LG, Osler TM, Mukamel DB, Meredith W, Dick AW. Expert consensus vs empirical estimation of injury severity: effect on quality measurement in trauma. Arch Surg. 2009; 144(4):326-32.

12. West SK, O’Mara MS, Spalding MC. Using clinical analysis to classify 30-day trauma readmissions. J Trauma Acute Care Surg. 2018;85(3):613-9.

13. Thompson AE, Anisimowicz Y, Miedema B, Hogg W, Wodchis WP, Aubrey-Bassler K. The influence of gender and other patient characteristics on health care-seeking behavior: a QUALICOPC study. BMC Fam Pract. 2016;17(38).

14. Parreco J, Buicko J, Cortolillo N, Narnias N, Rattan R. Risk factors and costs associated with nationwide nonelective readmission after trauma. J Trauma Acute Care Surg. 2017;83(1): 126-34.

15. Rooks MD, Johnston 3rd RB, Ensor CD, McIntosh B, James S. Injury patterns in recreational rock climbers. Am J Sports Med. 1995;23(6):683-5.

16. Kelly EA, Gonzalez LJ, Hutzler L, Konda S, Leucht P, Egol K. Readmissions are not what they seem: incidence and classification of 30-day readmissions after orthopedic trauma surgery. J Orthop Trauma. 2020;34(2):e72-6. 